\title{
Article \\ Design of a Cell Phone Lens-Based Miniature Microscope with Configurable Magnification Ratio
}

\author{
Xinjun Wan and Xuechen Tao *
}

check for

updates

Citation: Wan, X.; Tao, X. Design of a Cell Phone Lens-Based Miniature

Microscope with Configurable

Magnification Ratio. Appl. Sci. 2021

11, 3392. https://doi.org/10.3390/ app11083392

Academic Editor: Manuel Armada

Received: 12 March 2021

Accepted: 7 April 2021

Published: 9 April 2021

Publisher's Note: MDPI stays neutral with regard to jurisdictional claims in published maps and institutional affiliations.

Copyright: (C) 2021 by the authors Licensee MDPI, Basel, Switzerland. This article is an open access article distributed under the terms and conditions of the Creative Commons Attribution (CC BY) license (https:/ / creativecommons.org/licenses/by/ $4.0 /)$.
School of Optical-Electrical and Computer Engineering, University of Shanghai for Science and Technology, Shanghai 310000, China; xinjun.wan@usst.edu.cn

* Correspondence: 17621504336@163.com

\begin{abstract}
Application of cell-phone-based microscopes has been hindered by limitations such as inferior image quality, fixed magnification and inconvenient operation. In this paper, we propose a reverse cell phone lens-based miniature microscope with a configurable magnification ratio. By switching the objectives of three camera lens and applying the digital zooming function of the cell phone, a cell phone microscope is built with the continuously configurable magnification ratio between $0.8 \times-11.5 \times$. At the same time, the miniature microscope can capture high-quality microscopic images with a maximum resolution of up to $575 \mathrm{lp} / \mathrm{mm}$ and a maximum field of view (FOV) of up to $7213 \times 5443 \mu \mathrm{m}$. Furthermore, by moving the tube lens module of the microscope out of the cell phone body, the built miniature microscope is as compact as a $<20 \mathrm{~mm}$ side length cube, improving operational experience profoundly. The proposed scheme marks a big step forward in terms of the imaging performance and user operational convenience for cell phone microscopes.
\end{abstract}

Keywords: microscope; variable magnification; miniaturization

\section{Introduction}

Human beings have been supported by the microscope, which supported them in observing and recognizing things on a micro-scale, particularly in the aspect of biology and medicine, since it was invented [1]. To date, apart from making efforts to advance the performance of microscopes, researchers have also been focused on the miniaturization and cost reduction of microscopes in order to replace conventional bench-top instruments and meet the need for practical applications under the condition of resource-limited circumstances such as tele-medicine, teaching, and environmental monitoring [2].

Deriving from the impending demand for portability, small-scale size and cost compression, a great deal of study has been conducted on cell phones on the basis of the microscopic imaging system. Due to the popularization of cell phones and cellular networks worldwide, the operation of these kinds of cell phone microscopes is so uncomplicated that average users are able to carry out testing and upload the microscope images to the internet without the requirement of a complicated training process, which displays the remarkable advantage in terms of efficiency and accessibility in resource-limited situations. Some practicable approaches and techniques have been proposed, such as optofluidic microscopes [3], lens-free holographic microscopy [4] and cell phone microscopes using ordinary objectives [5,6], liquid lens, gel lens or simply a ball lens as the objective [7-9]. Among them, recently the cell phone microscope approach, by using a reversed cell phone camera lens as an object lens, demonstrates tremendous potential of the application with respect to imaging performances [10-12]. Such reversed camera lens-based miniature microscopes can conduct microscopic imaging with a field of view (FOV) up to mm level, with the resolution at the micrometer level having little distortion [13]. Moreover, the structure makes it easier to realize some super-resolution methods to further enhance imaging performance, such as fluorescence imaging [14,15] and computational imaging [12,16]. 
However, the practical applications of cell phone based miniature microscopes are still quite limited despite diversified proposed research. Unfortunately, miniature microscopes still suffer from a number of limitations [17]. The imaging performance of miniature microscopes is usually still inferior to ordinary bench-top systems. Reversed camera lensbased miniature microscopes can provide high-resolution and low-distortion microscope images [18], however, such miniature microscopes are of a fixed magnification ratio as reported, which is inconvenient compared with ordinary microscopes with switchable object lenses. Another problem is that it is not easy to handle cell phone miniature microscopes. Microscopic imaging requires precise control of the working distance between the microscope and sample by means of mechanism or shell [19], but it is usually inconvenient to fix and precisely adjust the large cell phone body. If complex adjusting structures are demanded, the miniature microscopes will lose advantages of portability and convenient deployment. Furthermore, in some applications, more than one miniature microscope is required to implement synchronous scanning and mosaic imaging when it comes to measuring the surface of large samples from the actual industry. It is difficult to accomplish with the reported designs to achieve the goal, as multiple cell phone cameras cannot be placed side by side closely and physically.

Given this, we propose a cell phone lens-based miniature microscope with a configurable magnification ratio, which still adopts the reversed cell phone camera lens concept, but puts forward two major methods of the melioration. Firstly, we propose to select different focal length object lenses from off-the-shelf cell phone camera lenses, thus achieving the goal of configurable values of FOV and resolution. There are numerous high-quality cell phone camera lenses commercially available nowadays, offering us a wide selection pool. Secondly, we suggest moving cell phone camera lenses outside of the cell phone body so as to realize an actual compact size microscope module, which is still connected to the cell phone. Therefore, the proposed cell phone microphone in this paper is expected to carry forward the advantages of low costs and direct access to the internet through the cell phone, simultaneously offering users capabilities of adjustable magnification ratios, really easy deployment and even array application in-field, which is of great importance for real application of cell phone microscopes.

\section{Materials and Methods}

\subsection{Principle of Miniature Cell Phone Microscope}

The function of the cell phone camera lens is to image a meter-scale scene onto a CMOS image sensor with a size of several millimeters (Figure 1A). At present, a cell phone is normally equipped with multiple cameras with diverse values of FOV, and the focal length of camera lenses varies within the range of $1-8 \mathrm{~mm}$. Such camera lenses are usually composed of 5-9 highly complex aspheric lenses. Therefore it is capable of high-quality imaging with minute aberration and field curvature and with a resolution power of up to $400-600 \mathrm{lp} / \mathrm{mm}$. Benefiting from the mass production of the cell phone industry, the cost of these precise camera lenses is fairly low compared with similar quality industrial camera lenses, making them attractive in the microscope domain.

The principle of the proposed miniature microscope is as follows (Figure 1B). The microscopic imaging system is usually a combination of the object lens, tube lens and camera sensor. Here, the cell phone camera serves as an accurate miniature component of the tube lens and CMOS sensor. The object lens of a microscope is realized with another cell phone camera lens, the direction of which is reversed. Then, the sample to be imaged is placed on the original CMOS sensor position, and the low field curvature feature of the cell phone camera lens makes it possible to image with a large FOV and high resolution. By combining the two cell phone lenses in this way, a microscope can be constructed with the following magnification ratio,

$$
M=f_{\text {tube }} / f_{\text {object }}
$$

where $f_{\text {tube }}$ and $f_{\text {object }}$ are focal length of tube lens and objective lens, respectively. 
(A)

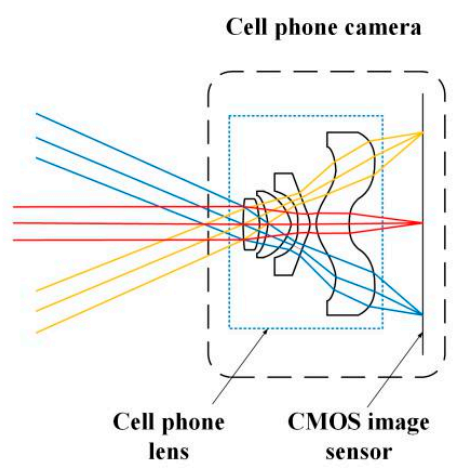

(B)

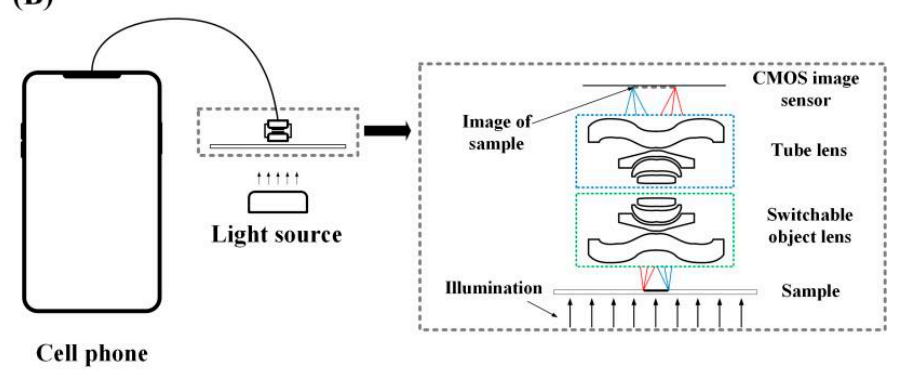

Figure 1. Principle of a miniature cell phone microscope. (A) Under normal circumstances, the cell phone lens can image a large field of view on a highly flat CMOS image sensor. This means that according to the principle of reversibility of light paths, high-quality microscopic imaging can be carried out when the cell phone lens is reversed. (B) Switchable reversed cell phone lenses are used as switchable objectives. They couple light from the sample through the tube lens and form the image of the sample on the CMOS image sensor. At last, the cell phone will process and display microscopic images. The microscope module is outside of the cell phone.

On this basis, we propose that the object lens can be a switchable camera lens module with a variable focal length so as to adjust the magnification of the microscope, which is a very desirable feature of the microscope. We can find a large pool of commercial cell phone camera lenses with different focal lengths, making this approach quite feasible. In addition, we suggest moving the miniature camera module (including tube lens and CMOS sensor) out of the cell phone, as shown in Figure 1B. The size of a miniature camera module is typically $10 \mathrm{~mm} \times 10 \mathrm{~mm} \times 8 \mathrm{~mm}$, while the cell phone size is typically about $120 \mathrm{~mm} \times 80 \mathrm{~mm} \times 10 \mathrm{~mm}$ by comparison. Therefore, compared with the previously reported cell phone microscope, the final size of the microscope module can be greatly reduced. The imaging operation can be greatly simplified, because the users only need to deal with a cube with a side length of $10 \mathrm{~mm}$.

Other advantages of the cell phone microscopes still exist in the proposed structure, including low costs and direct access to the Internet.

\subsection{Down-Selection of Different Effective Focal Length (EFL) Objectives}

According to the concept of the switchable object lens, we purchased a series of offthe-shelf cell phone camera modules and disassembled them to obtain a series of lenses with different focal lengths (Figure 2A). As accurate camera lens information is not easy to obtain, we firstly measured the focal lengths of lenses, as shown in Figure 2B. The fixed tube lens module is composed of a camera module (Figure 2C) with a known focal length $(\mathrm{EFL}=6 \mathrm{~mm})$ and a Sony IMX258 sensor (pixel size $=1.12 \mu \mathrm{m})$. The object lenses are connected with the module to image a $100 \mu \mathrm{m}$ wide line target (Edmund Optics). In this process, we designed an experimental system for precise adjustment of the working distance, building it with a 3D printer (Figure 2D). As for illumination, we utilized a simple 
LED diffuse backlight in the transmission approach. The results of the imaging test are presented in Figure 3.

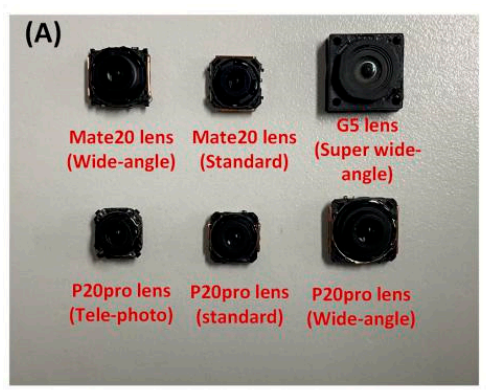

(C)

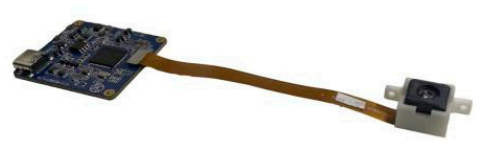

(B)

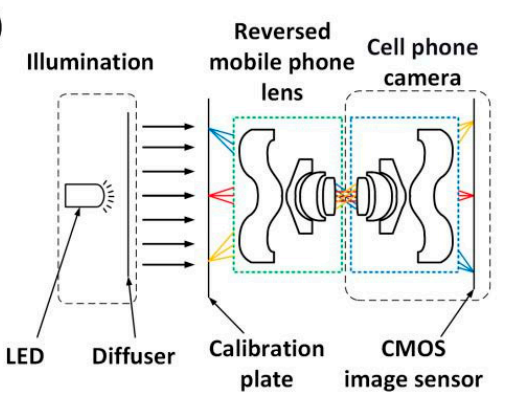

(D)

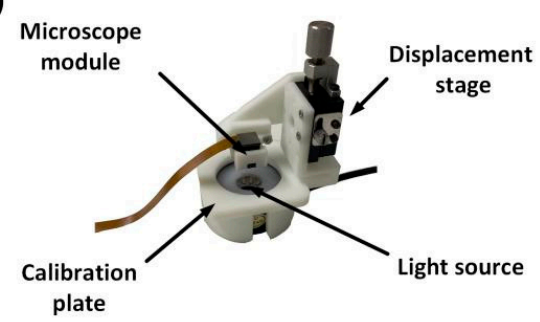

Figure 2. Measurement of selected off-shelf cell phone camera lens. (A) The six cell phone lenses with distinct focal lengths are all obtained from disassembled cell phones of Huawei P20pro, Mate20, and LG G5. (B) The focal length measurement device consists of a diffused light source, calibration plate, the lens to be measured and a camera module with known parameters. (C) The image of the tube lens camera module with known focal length and Sony IMX258 sensor. (D) The experimental system consists of a microscope module, displacement stage, calibration and light source. The frame is produced by $3 \mathrm{D}$ printing.
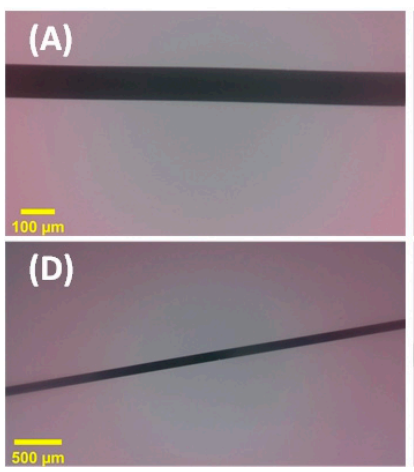
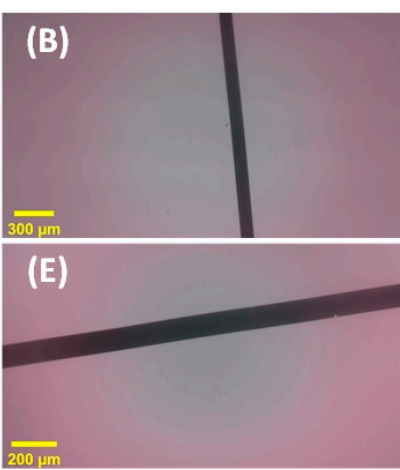
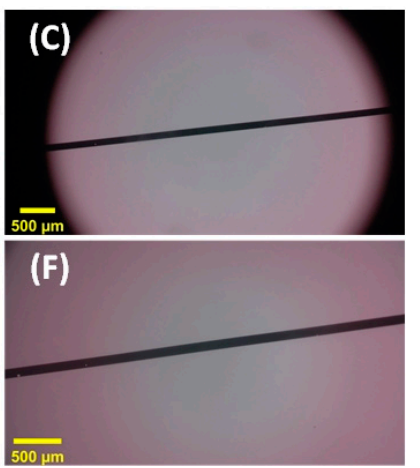

Figure 3. Photos of $100 \mu \mathrm{m}$ wide line target obtained by the tube lens (EFL $=6 \mathrm{~mm}$ ) matched with different cell phone camera lenses. (A-F) The pictures correspond to different object lenses in turn, which are LG G5 super wide-angle lens, P20pro wide-angle lens, P20pro tele-photo lens, P20pro standard lens, Mate20 wide-angle lens and Mate20 standard lens.

The imaging test results of the six dismantled lenses are shown in Figure 3. The magnification ratio can be determined according to the line width (in pixels) in the image, the pixel size on the sensor and the nominal line width of the sample. The focal length of the dismantled lenses can be calculated based on Equation (1). As shown in Table 1, by selecting different lens modules, we can realize the switching range of magnification of $\sim 3.9 \times$. In the following experiments, we selected three lenses as the objectives in the miniature microscope, namely LG G5 super wide-angle lens (FL = $1.5 \mathrm{~mm}$ ), Huawei P20pro standard lens ( $\mathrm{FL}=6 \mathrm{~mm}$ ) and P20pro wide-angle lens ( $\mathrm{FL}=4 \mathrm{~mm})$. The larger focal length lens (Huawei P20pro tele-photo) was not selected for aperture mismatch reasons, which will be discussed in the following part. 
Table 1. Measurement result of magnification ratio and calculated focal length.

\begin{tabular}{cccccc}
\hline Camera Lens Source & Magnification Ratio & $\begin{array}{c}\text { Focal Length } \\
(\mathbf{m m})\end{array}$ & $\begin{array}{c}\text { Camera Lens Source } \\
\text { Magnification Ratio }\end{array}$ & $\begin{array}{c}\text { Focal Length } \\
(\mathbf{m m})\end{array}$ \\
\hline $\begin{array}{c}\text { Mate20 } \\
\text { (Wide-angle) }\end{array}$ & 2.4 & 2.5 & $\begin{array}{c}\text { P20pro } \\
\text { (Wide-angle) }\end{array}$ & 1.5 & 4.0 \\
\hline $\begin{array}{c}\text { Mate20 } \\
\text { (Standard) }\end{array}$ & 1.2 & 5.0 & $\begin{array}{c}\text { P20pro } \\
\text { (Tele-photo) }\end{array}$ & 0.75 & 8.0 \\
\hline $\begin{array}{c}\text { G5 } \\
\text { (Super wide-angle) }\end{array}$ & 3.9 & 1.5 & $\begin{array}{c}\text { P20pro } \\
\text { (Standard) }\end{array}$ & 1.0 & 6.0 \\
\hline
\end{tabular}

\subsection{Assembly of Miniature Microscope with Switchable Magnification Ratios}

We also suggest removing the tube lens module from the cell phone to form a truly compact cell phone microscope. In the experiment, we dismantled a second-hand Huawei Honor 7 cell phone and took its main back cameras with a $20 \mathrm{M}$ pixel out of the package, and connected them with a flexible cable (Figure 4A). This camera, with a known focal length $(\mathrm{EFL}=4.62 \mathrm{~mm}$ ), is equipped with a Sony IMX230 sensor (pixel size $=1.12 \mu \mathrm{m}$ ). Then, a compact fixture is designed to accommodate the main camera module (the tube lens) and the selected camera lens module (the objective module), as shown in Figure 4B. The fixture can be installed on a 3D printed threaded ring and the distance between the objective and the sample can be adjusted by rotating the ring to find the best focusing position. The side length of the whole fixture is only about $15 \mathrm{~mm}$. A commercial LED diffuse reflection backlight is used as the transmission light source (Figure 4C), which can be replaced with other available light sources in practical applications. Users can control focusing adjustment and capture microscopic images through cell phone camera software.
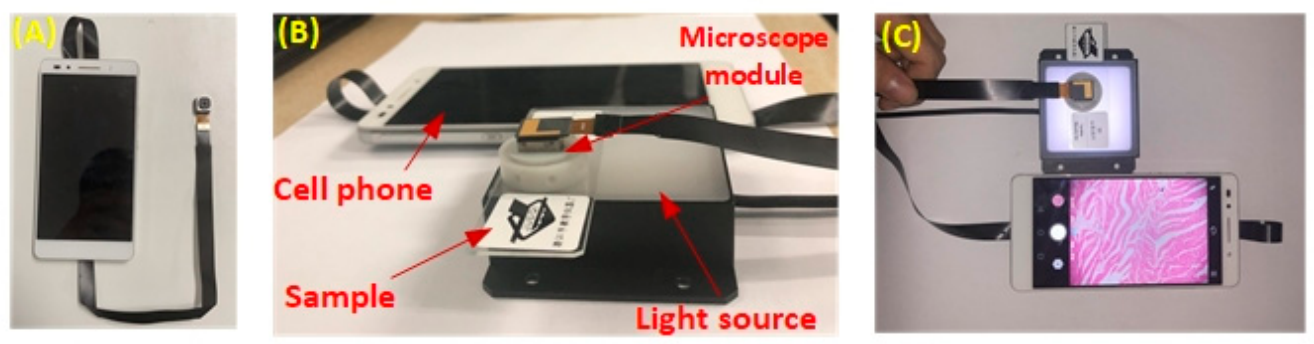

Figure 4. Fabricated miniature cell phone microscope. (A) After modification, the cell phone camera can be led out of the cell phone by a flexible cable. $(\mathbf{B}, \mathbf{C})$ Image of the miniature microscope configuration and working scene.

The constructed miniature cell phone microscope has several merits compared with the former reported systems. Firstly, the whole system is highly portable, especially the microscope module. Secondly, the operation of the miniature microscope is really simple, even in the field, which is due to the compact size. There is no need for complicated focal length adjustment rigs. Thirdly, the FOV can be easily switched by shifting three objectives whose diameters were made equal by 3D printed adapters. Switchable magnification enables the proposed microscope to observe the sample, select region of interest (ROI) to enlarge the magnification and review with a higher resolution, similar to the operation of bench-top microscopes. Finally, the image quality of the microscope matches that of the bench-top systems, since all the lenses utilize the high industrial quality of the cell phones.

We noticed another advantage of the cell phone microscope. Modern cell phones are all equipped with digital zooming functions (Figure 5) and advanced build-in image processing functions. The largest digital zooming ratio of Huawei Honor 7 is $\sim 4 \times$, thus giving users more flexibility in continuous magnification adjustment. According to our tests, compared with the zero zoom condition, the digital zoom images provide higher 
image resolution. Therefore, the digital zooming of cell phones has brought real benefits to microscope performance.

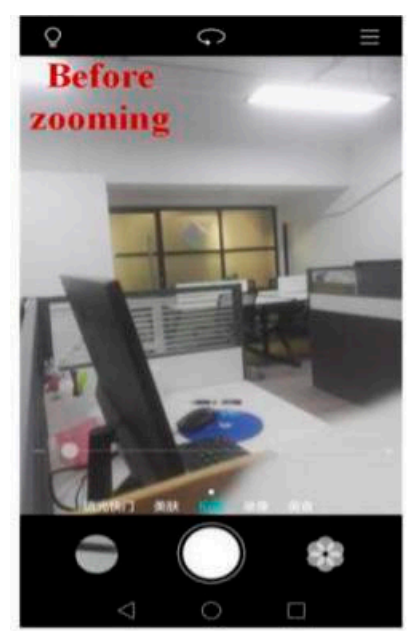

(A)

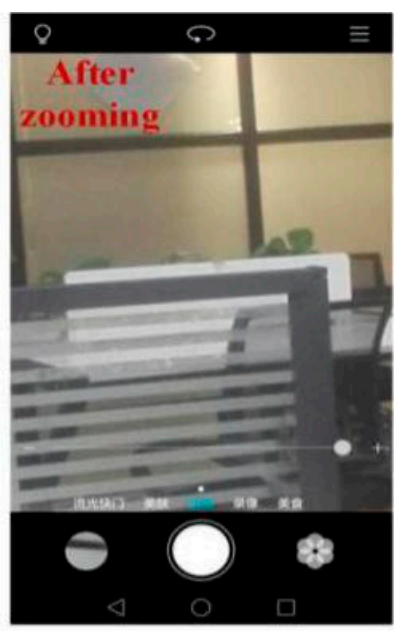

(B)

Figure 5. Two mobile photos taken in normal environment. The zoom function of the cell phone camera can be operated manually in the interface. (A) Photo before zooming. (B) Photo after zooming.

\section{Results}

\subsection{Microscopic Imaging Performance Tests}

Firstly, we evaluated the imaging performance of the constructed miniature cell phone microscope by imaging a resolution test chart(USAF-1951 standard resolution target), through which the values of FOV and resolution at three magnification ratio settings could be determined. The captured images exhibit high clarity and little distortion, which are comparable to the bench-top systems (Figure 6). All the images are obtained directly from the cell phone without any post-treatment. When the zooming function of the cell phones is not applied, the diagonal values of the FOV at three magnification ratios are $2.7 \mathrm{~mm}, 6.0 \mathrm{~mm}$ and $9.0 \mathrm{~mm}$, corresponding to the magnification ratios of $3 \times, 1.2 \times$ and $0.8 \times$, respectively. When the digital zooming of the cell phones is applied and the sample is re-focused, the FOV is shrunken, yet the image reveals more detailed features. We evaluated the image resolution based on the intensity contrast of the line profile between the horizontal and vertical directions. The microscope can distinguish the features of $575 \mathrm{lp} / \mathrm{mm}, 322 \mathrm{lp} / \mathrm{mm}$ and $203 \mathrm{lp} / \mathrm{mm}$ when the three lenses are used, respectively. If we take the FOV into consideration, we can find that the constructed miniature microscopes outperform some bench-top systems in terms of their comprehensive imaging performance, which makes them quite attractive in the applications of large sample screening and microscopic reviewing. The low cost and compact size add more advantages to the proposed miniature microscope.

\subsection{Real Sample Imaging Test}

We used the miniature microscope to conduct imaging tests of real samples, which included a plant terminal bud slice, a kidney vertical slice and a fiber connective tissue slice. Such samples are commercially available for educational purposes. With the help of the miniature microscope, we can take impressive microscopic images of the samples (Figure 7). We can find that the switching of the magnification ratio proposed here has important value in the process of sample imaging. Take the imaging of the plant terminal bud slide as an example. The $1.2 \times$ mag ratio image gives users an overall view of the bud sample and finds the region of interest(ROI), and digital zooming offers a $\sim 4 \times$ larger image of the central region with more details. However, users can not observe the cell nucleus clearly since the resolution using this lens module is only about $400 \mathrm{lp} / \mathrm{mm}$. By shifting 
to the G5 lens module objective, users can obtain the $3 \times$ image before zooming and the $11.5 \times$ image after zooming. Users are now able to discriminate the internal structures of the cells with micron-level resolution. The workflow is similar to that of bench-top systems. Therefore, users may feel it easier to learn and practice the miniature microscope. The captured images are already colored images, which indicate that the proposed systems have great potential for histology sample imaging.

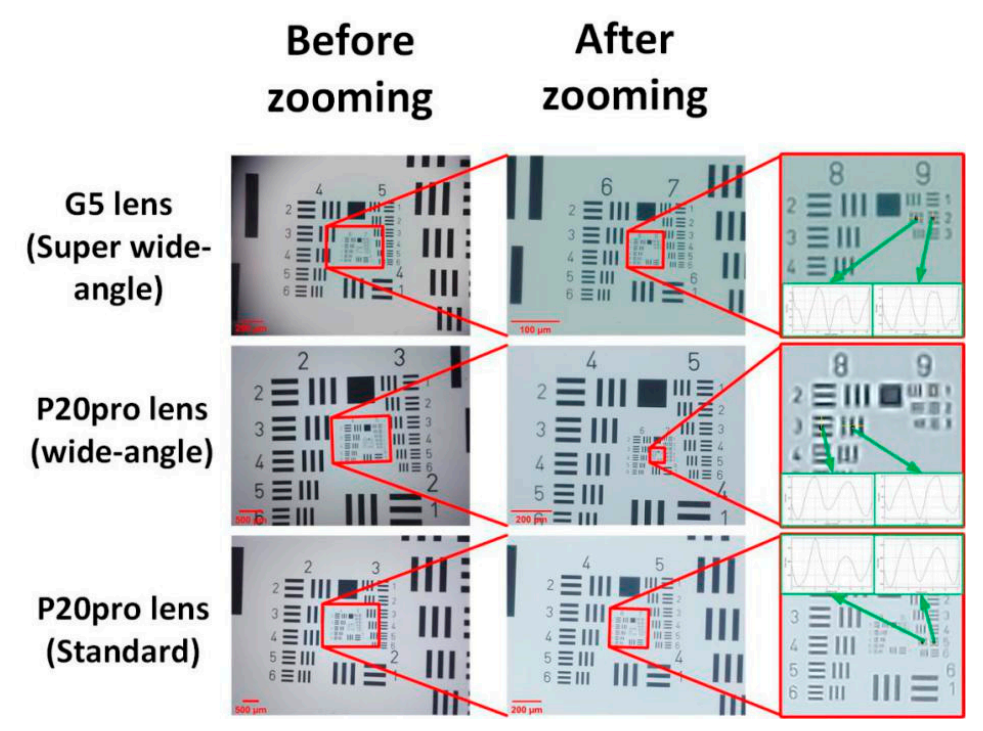

Figure 6. Evaluation of imaging performance of the built microscope. LG G5 super wide-angle lens, P20pro wide-angle lens and P20pro standard lens are taken as targets to take photos of resolution chart. When the zooming function of the cell phones is not applied, the diagonal values of the field of view (FOV) at the three magnification ratios are $2.7 \mathrm{~mm}, 6.0 \mathrm{~mm}, 9.0 \mathrm{~mm}$, which correspond to the magnification ratios of $3 \times, 1.2 \times$ and $0.8 \times$, respectively. The FOV is reduced when using the digital zoom of the cell phones, but the microscope can distinguish the features of $575 \mathrm{lp} / \mathrm{mm}, 322 \mathrm{lp} / \mathrm{mm}$ and $203 \mathrm{lp} / \mathrm{mm}$, respectively.

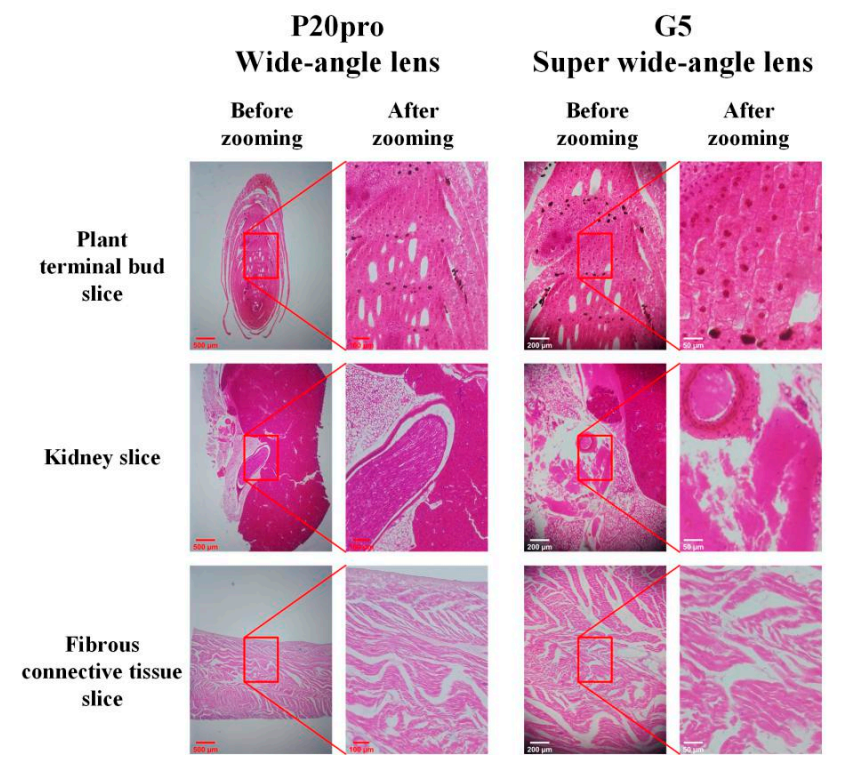

Figure 7. Imaging tests of real biological samples. Sample images of a plant terminal bud slice, a kidney slice and a fibrous connective tissue slice are obtained with the built microscope using the P20pro wide-angle lens and the LG G5 Super wide-angle lens as objectives. We also used the zoom function in the imaging process. 


\section{Discussions}

The imaging tests exhibit that the proposed miniature microscope can provide micronlevel resolution and large FOV microscopic imaging with minute cost and high portability. Next, the limiting factors of miniature cell phone microscopes are discussed.

The first problem is whether the FOV of the miniature microscope can be enlarged. According to Equation (1), the longer the focal length of the object lens switched to, the larger the FOV for the proposed microscope is. However, for the cell phone tele-photo lens, the increase of focal length brings with it a smaller angle of imaging, since they are usually designed for the fixed size CMOS sensor. If a long focal length tele-photo cell phone lens (for example, P20pro tele-photo, $\mathrm{EFL}=8 \mathrm{~mm}$ ) is used, we find the resulting image is effective only in the central circular region, which is decided by the maximum imaging angle of the tele-photo lens (Figure 8). Compared with Figure 6, we can see that the magnification ratio decreases as expected, and the effective FOV also decreases. Therefore, we abandoned the tele-photo lens as the objective. If users really demand an even larger FOV, a specially designed tele-photo lens with larger imaging angle may be required, which may increase the cost of the total system.

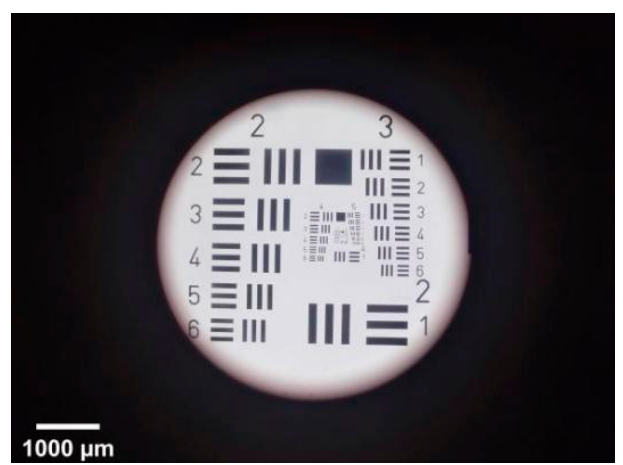

Figure 8. Picture of resolution test chart using P20pro tele-photo lens ( $E F L=8 \mathrm{~mm}$ ). Theoretically, when the longer focal length lens is used as an objective lens, the magnification will be reduced and the FOV will become larger. In practice, the FOV may be limited to a smaller circle by the objective lens imaging angle limitation.

The proposed miniature microscope is formed by connecting two cell phone lenses. The mismatch between the position and the size of the two lens apertures would naturally have a negative impact on the imaging quality of the micro-imaging system. In Table 2, the resolution is evaluated based on the central part of the image, where the influence of aperture mismatch is minimal. It can be expected that vignetting may occur in the off-axis field of view due to aperture. From previous results, we can find that the corner area of the image is obviously darkened at a low magnification ratio (Figure 6). Resolution is also influenced by this factor. When we shifted the 8th and 9th group lines of the resolution test chart from middle to boundary of the field of view, the aberration aggregation can be observed and the resolution capability gets worse, as the resolvable 9-1 lines become contrast-less in the corner of the image (Figure 9). For some applications, the aberration on the edge would further weaken the available field of view, even though the imaging amount in the center is acceptable, especially when scanning or stitching is needed. By shortening the distance between the two lenses in the fixture, the influence of aperture mismatch may be reduced. In addition, when the objective lens is selected, the cell phone lens with a larger aperture is preferred.

Other limiting factors also include color non-uniformity of the microscope image, short working distance, etc. However, despite all these limitations, the proposed miniature cell phone microscope with switchable magnification marks the improvement of imaging performance and user operational acceptance. 
Table 2. Measurement result of the magnification ratio, FOV and resolution.

\begin{tabular}{|c|c|c|c|c|c|}
\hline & \multicolumn{2}{|c|}{ Before Zooming } & \multicolumn{2}{|c|}{ After Zooming } & \multirow{2}{*}{$\begin{array}{l}\text { Highest Resolution } \\
(1 \mathrm{p} / \mathrm{mm})\end{array}$} \\
\hline & Magnification Ratio & FOV/um & Magnification Ratio & FOV/um & \\
\hline $\begin{array}{c}\text { G5 } \\
\text { (Super wide-angle) }\end{array}$ & 3.0 & $2137 \times 1613$ & 11.5 & $505 \times 381$ & 574.7 \\
\hline $\begin{array}{c}\text { P20pro } \\
\text { (Wide-angle) }\end{array}$ & 1.2 & $4809 \times 3629$ & 4.9 & $1178 \times 889$ & 406.4 \\
\hline $\begin{array}{c}\text { P20pro } \\
\text { (Standard) }\end{array}$ & 0.8 & $7213 \times 5443$ & 3.3 & $1749 \times 1320$ & 228.1 \\
\hline
\end{tabular}

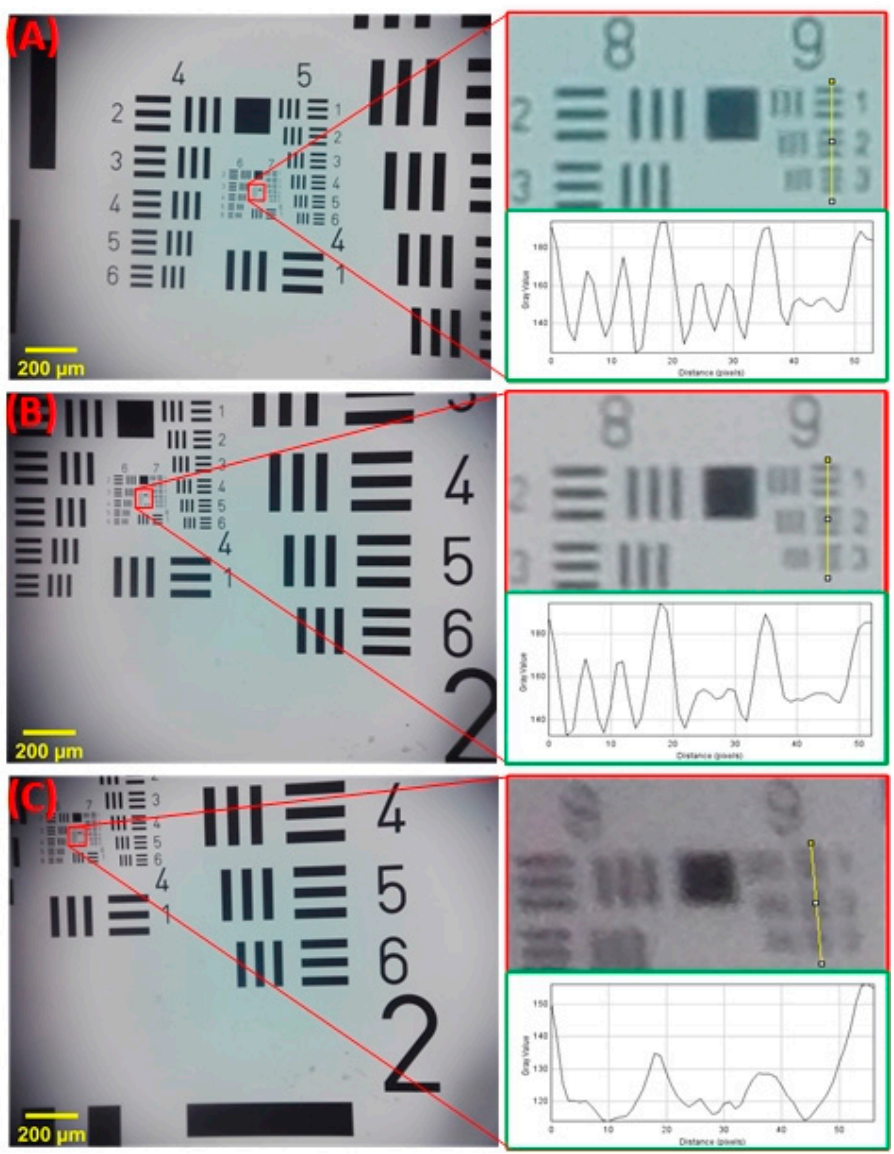

Figure 9. Three sets of images taken of the resolution test chart as it moves from the FOV center to boundary. (A) Lines of $575 \mathrm{lp} / \mathrm{mm}$ can be clearly distinguished when it is at the center of the FOV. (B) The $575 \mathrm{lp} / \mathrm{mm}$ contrast is reduced when the resolution test chart is moved outward from the center. (C) The $575 \mathrm{lp} / \mathrm{mm}$ features are blurred at the corner of the FOV due to vignetting and optical aperture mismatch.

More importantly, the proposed concept can be used as a miniature platform for various interesting micro-imaging research applications. In the cell culture applications, the real-time microscopic wireless observation can be realized (Figure 10A). The compact size of the microscope module makes it possible to be placed inside the temperature-controlled cell culturing tank, and the captured images can be transferred via the cell phone in a real-time manner. In addition, the low cost of the miniature microscope makes it possible to deploy such a module array to monitor an array of cell culturing plates simultaneously. This research is already in progress and has aroused great interest. In this paper, we have demonstrated the maximum magnification of $11.5 \times$ and a $\sim 600 \mathrm{lp} / \mathrm{mm}$ resolution power, which is already one of the best results for such kinds of cell phone microscopes. However, 
the higher resolution is still expected to gain more application possibilities. We have already been investigating the schemes of combining the proposed miniature microscope with other principles, such as total internal reflection imaging (Figure 10B), structured light imaging (Figure 10C), etc. The proposed scheme enjoys a relatively large FOV in the flat field, making it suitable for carrying out such super-resolution methods. In the near future, advanced fluorescence microscopy with low costs and high resolution will be completely possible. Other interesting applications may include plant leaf or water particle real-time monitoring, etc. In many applications, the $\sim 1-\mathrm{mm}$ long working distance of the proposed microscope is acceptable and as long as this is satisfied, many application schemes can be imagined and designed on the merit of its compact size, reasonable resolution power, large FOV, tale-communication ability and low cost.

(A)

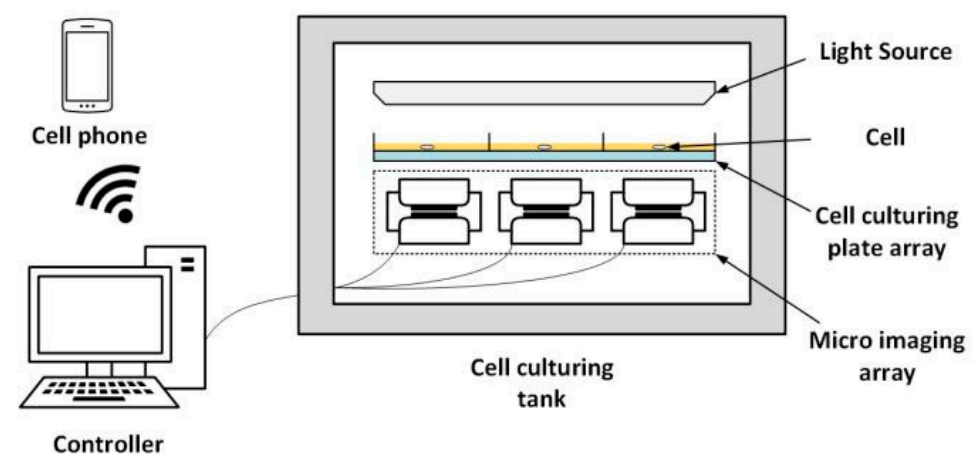

(B)
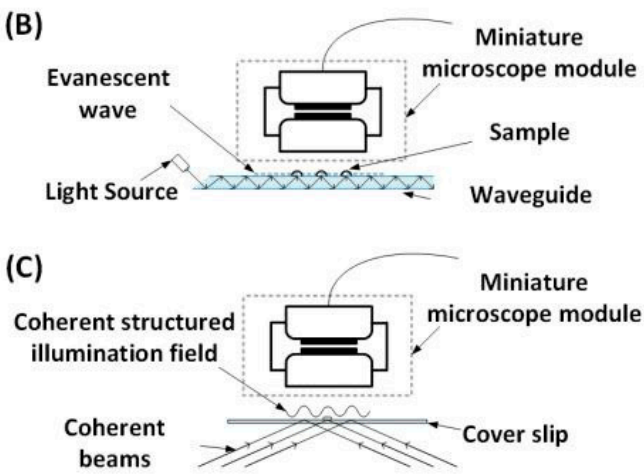

Figure 10. Potential applications based on the proposed miniature microscope. (A) Real-time microscopic wireless observation of the enclosed cell culturing process. (B) Scheme of a low-cost total internal reflection miniature microscope. (C) Scheme of a low-cost structured illumination miniature microscope for enhanced resolution.

\section{Conclusions}

Cell phone microscopes have been widely researched, the applications of which are still limited by the following factors, including inferior image quality, fixed magnification, inconvenient operation, etc. In this paper, we proposed a cell phone lens-based miniature microscope with a configurable magnification ratio. Inheriting the advantages of low costs and direct access to the internet via cell phone, the proposed miniature microscope simultaneously provides users with the ability of adjustable magnification ratios, truly easy deployment and even an array of applications in the field. These improvements are significant for the field applications of cell phone microscopes.

On the basis of the proposed scheme, by switching three camera lens objectives and applying the digital zooming function of the cell phones, a set of miniature microscopes with magnification ratios continuously configurable among $0.8 \times-11.5 \times$ was built. The imaging tests exhibit that the proposed miniature microscope can provide high-quality microscopic imaging, with a maximum resolution of up to $575 \mathrm{lp} / \mathrm{mm}$ and a maximum FOV of up to $7213 \times 5443 \mu \mathrm{m}$. In addition, by moving the tube lens module of the cell phone microscope out of the cell phone body, the built miniature microscope is as compact as a cube with a side length of $15 \mathrm{~mm}$, profoundly advancing the operational experience. We believe that the proposed scheme has addressed the mentioned limitations of the cell phone-based microscopes and users may find it easier to learn and practice with such miniature microscopes. With the launch of more powerful cell phone cameras these days, we can foresee that more powerful miniature cell phone microscopes will be produced and put into use.

Author Contributions: Investigation, X.W. and X.T.; methodology, X.W. and X.T.; resources, X.W.; supervision, X.W.; validation, X.W. and X.T.; writing-original draft, X.T.; writing-review and editing, X.W. and X.T. All authors have read and agreed to the published version of the manuscript. 
Funding: This research was funded by the National Natural Science Foundation of China, grant number 61505107 and by the Shanghai Science and Technology Innovation Action Plan, grant number 19511104600.

Institutional Review Board Statement: Not applicable.

Informed Consent Statement: Not applicable.

Data Availability Statement: The data used to support the findings of this study are included within the article.

Conflicts of Interest: The authors declare no conflict of interest.

\section{References}

1. Schiffer, M.B.; Miller, A.R. The Material Life of Human Beings: Artifacts, Behavior, and Communication; Psychology Press: Hove, UK, 1999.

2. Petti, C.A.; Polage, C.R.; Quinn, T.C.; Ronald, A.R.; Sande, M.A. Laboratory medicine in Africa: A barrier to effective health care. Clin. Infect. Dis. 2006, 42, 377-382. [CrossRef] [PubMed]

3. Zheng, G.; Lee, S.A.; Yang, S.; Yang, C. Sub-pixel resolving optofluidic microscope for on-chip cell imaging. Lab. Chip 2010, 10, 3125-3129. [CrossRef] [PubMed]

4. Bishara, W.; Su, T.W.; Coskun, A.F.; Ozcan, A. Lensfree on-chip microscopy over a wide field-of-view using pixel super-resolution. Opt. Express 2010, 18, 11181-11191. [CrossRef] [PubMed]

5. Skandarajah, A.; Reber, C.D.; Switz, N.A.; Fletcher, D.A. Quantitative imaging with a mobile phone microscope. PLoS ONE 2017, 9, e96906. [CrossRef] [PubMed]

6. Cai, F.; Wang, T.; Lu, W.; Zhang, X. High-resolution mobile bio-microscope with smartphone telephoto camera lens. Optik 2020, 207. [CrossRef]

7. Breslauer, D.N.; Maamari, R.N.; Switz, N.A.; Lam, W.A.; Fletcher, D.A. Mobile phone based clinical microscopy for global health applications. PLoS ONE 2009, 4, e6320. [CrossRef] [PubMed]

8. Smith, Z.J.; Chu, K.; Espenson, A.R.; Rahimzadeh, M.; Gryshuk, A.; Molinaro, M.; Dwyre, D.M.; Lane, S.; Matthews, D.; Wachsmann-Hogiu, S. Cell-phone-based platform for biomedical device development and education applications. PLoS ONE 2011, 6, e17150. [CrossRef] [PubMed]

9. Switz, N.A.; D'Ambrosio, M.V.; Fletcher, D.A. Low-cost mobile phone microscopy with a reversed mobile phone camera lens. PLoS ONE 2014, 9, e95330. [CrossRef] [PubMed]

10. McKay, G.N.; Mohan, N.; Butterworth, I.; Bourquard, A.; Sánchez-Ferro, Á.; Castro-González, C.; Durr, N.J. Visualization of blood cell contrast in nailfold capillaries with high-speed reverse lens mobile phone microscopy. Biomed. Opt. Express 2020, 11, 2268-2276. [CrossRef] [PubMed]

11. Dong, S.; Guo, K.; Nanda, P.; Shiradkar, R.; Zheng, G. FPscope: A field-portable high-resolution microscope using a cellphone lens. Biomed. Opt. Express 2014, 5, 3305-3310. [CrossRef] [PubMed]

12. Kim, J.-H.; Joo, H.-G.; Kim, T.-H.; Ju, Y.-G. A smartphone-based fluorescence microscope utilizing an external phone camera lens module. BioChip J. 2015, 9, 285-292. [CrossRef]

13. Kim, K.M.; Choe, S.-H.; Ryu, J.-M.; Choi, H. Computation of Analytical Zoom Locus Using Padé Approximation. Mathematics 2020, 8, 581. [CrossRef]

14. Chen, X.; Yu, L.; Kang, Q.; Sun, Y.; Huang, Y.; Shen, D. A smartphone-based absorbance device extended to ultraviolet (365 nm) and near infrared $(780 \mathrm{~nm})$ regions using ratiometric fluorescence measurement. Microchem. J. 2021, 164, 105978. [CrossRef]

15. Goenka, C.; Lewis, W.; Chevres-Fernández, L.R.; Ortega-Martínez, A.; Ibarra-Silva, E.; Williams, M.; Franco, W. Mobile phonebased UV fluorescence microscopy for the identification of fungal pathogens. Lasers Surg. Med. 2019, 51, 201-207. [CrossRef] [PubMed]

16. Diederich, B.; Wartmann, R.; Schadwinkel, H.; Heintzmann, R. Using machine-learning to optimize phase contrast in a low-cost cellphone microscope. PLoS ONE 2018, 13, e0192937. [CrossRef] [PubMed]

17. Helmchen, F.; Fee, M.S.; Tank, D.W.; Denk, W. A miniature head-mounted two-photon microscope: High-resolution brain imaging in freely moving animals. Neuron 2001, 31, 903-912. [CrossRef]

18. Rolland, J.P.; Fuchs, H. Optical versus video see-through head-mounted displays in medical visualization. Presence Teleoper. Virtual Environ. 2000, 9, 287-309. [CrossRef]

19. Di Febo, R.; Casas, L.; Antonini, A. A smartphone-based petrographic microscope. Microsc. Res. Tech. 2021. [CrossRef] [PubMed] 